\title{
The Role of Endoplasmic Reticulum Stress in the Development of Fibrosis in Crohn's Disease
}

\author{
Chao Li* \\ Department of Medicine, Division of Gastroenterology, Hepatology \& Nutrition, Virginia Commonwealth University, \\ 1220 E Broad Street, P.O. BOX 980341, Richmond, VA 23298-0341, USA
}

\begin{abstract}
When cells are subject to endoplasmic reticulum (ER) stress due to inflammation, inadequate nutrition or infection, a characteristic environment of glucose starvation, acidosis and hypoxia is created. All the conditions cited contribute to ER stress as well as the unfolded protein response (UPR). The UPR is active in a variety of human tumor types. Depending on the severity of ER stress, the UPR can exert a cytoprotective function by resolving the misfolded or unfolded protein, further reducing the ER protein load in mild stress, or by sending signal to cells to undergo cell death by apoptosis in the severe condition. Recent studies suggest that the glucose-regulated protein (GRP)78, or immunoglobulin heavy chain binding protein (BiP), not only confers an advantage to cell survival through an anti-apoptotic function but also may improve cell proliferation and angiogenesis. Understanding how the UPR can induce adaptation to chronic stress instead of apoptosis in cells will be of invaluable significance toward finding a cure for ER stress-associated diseases. This review covers what is known about the adaptive responses of cells when facing ER stress and how these information may lead to discoveries of novel treatments for various related disorders. Studies have suggested that ER stress promotes tissue remodeling under a variety of conditions and through several mechanisms, including activation of apoptosis, epithelialmesenchymal transition, and enhanced inflammatory response. This review also focuses on the major cell components in the gut, primarily epithelial cells and mesenchymal cells, for which a myriad of evidence suggests that sustained ER stress causes epithelial cell damage along with resultant mucosal barrier dysfunction, stimulates

Keywords: Intestinal fibrosis; ER stress; Mesenchymal cell; Inflammatory bowel disease; Apoptosis.

Abbreviations: ASK1, apoptosis signal-regulating kinase 1; ATF, activating transcription factor; BAD, Bcl-2 antagonist of cell death; BAK, Bcl-2 homologous antagonist/killer; Bax, Bcl2-associated X protein; Bcl-2, B cell leukemia 2; Bim, Bcl2-interacting mediator of cell death; $\mathrm{BiP}$, binding protein; bZiP, basic leucine zipper; C/EBP, CCAAT/enhancer-binding protein; CHOP, C/EBP homologous protein; DR5, death receptor 5; ECM, extracellular matrix; eIF2 a, a-subunit of eukaryotic translational initiation factor 2; EMT, Epithelial-mesenchymal transition; ER, endoplasmic reticulum; ERAD, ER-associated degradation; ERO1, ER oxidase; ERSE, ER stress response element; GADD, growth arrest and DNA damage; GRP, glucose-regulated protein; HSC, hepatic stellate cell; HSP, heat shock protein; IBD, inflammatory bowel disease; IEC, intestinal epithelial cells; IL, interleukin; IRE1, inositol requirement 1; JNK, Jun N-terminal kinase; M2, activated macrophage phenotype; MAPKKK, mitogen-activated protein kinase kinase kinase; miR, microRNA; MMP, matrix metalloproteinase; MOMP, mitochondrial outer membrane permeabilization; NOXA, neutrophil NADPH oxidase factor; PDI, protein disulfide isomerase; PERK, PKRlike endoplasmic reticulum kinase; PP, protein phosphatase; PUMA, p53 up-regulated modulator of apoptosis; RCAN1, regulator of calcineurin 1; ROS, reactive oxygen species; S1P, site 1 protease; S2P, site 2 protease; SMA, smooth muscle actin; TGF,

mesenchymal cell differentiation, and induces myofibroblasts activation and their secretion of excess extracellular protein leading to matrix collagen-rich tissue formation. Several key questions are still not answered by the fibrosis research and are discussed in this review: for example, the mechanisms of ER stress induction and the specific signaling pathway(s) activated by which UPR leads to development of organ fibrosis, or how to maintain ER stress at a basal level instead of exacerbating its physiological role that is otherwise necessary to maintain intracellular homeostasis. Ultimately, further investigations are needed to bridge the gap between our current understanding of ER stress mechanisms and to identify efficient anti-fibrotic therapeutic regimens.
\end{abstract} transforming growth factor; TIMP, tissue inhibitor of matrix metalloproteinase; TNF, tumor necrosis factor; TRAF2, TNF receptor-associated factor 2; UPR, unfolded protein response; XBP1, $\mathrm{x}$-box binding protein 1 ; ZO-1, zonula occludens-1.

Received: March 18, 2018; Revised: May 30, 2018; Accepted: June 05, 2018

"Correspondence to: Chao Li, Department of Medicine, Division of Gastroenterology, Hepatology \& Nutrition, Virginia Commonwealth University, 1220 E Broad Street, P.O. BOX 980341, Richmond, VA 23298-0341, USA. Tel: 804-628-5400; E-mail: chao.li@vcuhealth.org

How to cite this article: Li C. The Role of Endoplasmic Reticulum Stress in the Development of Fibrosis in Crohn's Disease. Exploratory Research and Hypothesis in Medicine 2018;3(2):33-41. doi: 10.14218/ERHM.2018.00003

\section{Introduction}

The endoplasmic reticulum (ER) is well known as a fundamental cellular compartment required for synthesis, assembly, modification and trafficking of secretary and membrane proteins. ${ }^{1-3}$ Innumerable environmental stimuli and pathological conditions alter 
protein-folding reactions in the ER through multiple mechanisms, which include hypoxia, depletion of ER calcium, alteration in the redox status, energy (glucose) starvation, viral infection, gene mutations, altered post-translational modification, hypoglycemia, and protein inclusion bodies. ${ }^{1-3}$

Accumulation of unfolded or misfolded proteins that emerge in the ER lead to an evolutionarily conserved series of signaling pathways, termed the unfolded protein response (UPR). ${ }^{1-3}$ As a consequence of UPR activation, ER-associated degradation (ERAD) happens by removal of misfolded proteins or by enhanced folding and transport of nascent proteins such that they avoid misfolding in the ER. ${ }^{4}$ Conversely, if ER stress is severe and cannot resolve the protein folding defect, the UPR can induce cell death through the activation of apoptotic signaling pathways directed particularly by the ER, as well as coupling with the mitochondrial signaling pathways. ${ }^{1-3}$

However, this continuing activation can be accommodated with suppression of UPR-dependent apoptosis in mesenchymal cells that contribute to fibrosis. One possibility for inhibition of apoptosis and favoring adaptation under such circumstances is the stability of mRNA and protein of adaptive genes, such as the ER chaperones glucose-regulated protein (GRP)78 and GRP94, and the instability of mRNA and protein of transcription factor CAAT/ enhancer binding protein $(\mathrm{C} / \mathrm{EBP})$ homologous protein, $\mathrm{C} / \mathrm{EBP}$ homologous protein $(\mathrm{CHOP})$ and growth arrest and DNA damage (GADD) 34. ${ }^{2}$

Here, I first review the biochemical events related to the ER stress and the major molecules involved in the UPR. Then, I discuss the association between ER stress and apoptosis within different type of cells as well as certain signaling pathways reported in previous studies. Finally, the future directions and potential solutions are addressed for the therapeutic potential of ER stresstargeted therapy for fibrotic disease, particularly intestinal fibrosis.

\section{ER stress concept}

In eukaryotic ells, ER has a membranous labyrinth network and is an ultimate perinuclear organelle where cell surface and secreted proteins can be synthesized and maintained with high fidelity through the assistance of molecular chaperones, such as GRP78, and folding enzymes, such as protein disulfide isomerases (PDIs). ${ }^{1-5}$ Only correctly folded proteins can be transported to the Golgi apparatus. Unfolded or misfolded proteins are retained in the ER, further inverse translocated from the ER lumen to the cytosol by the machinery of ERAD, and usually degraded by the $26 \mathrm{~S}$ proteasome. ${ }^{2,6}$

An imbalance between the ER and the capacity of the folding apparatus and ERAD machinery will trigger a cytoprotective signal transduction pathway called the UPR and set four main responses in motion:1,2,4 (1) translational attenuation, which prevents excessive accumulation of unfolded proteins; (2) up-regulation of ER chaperones and folding enzymes, which increases the protein folding capacity; (3) enhanced ERAD of misfolded proteins, which strengthen ERAD ability to clear unfolded proteins and send them to the cytoplasm for proteasome involved degradation; and (4) induction of apoptosis, which happens when the unfolded protein in the ER is protracted or overwhelming and the adaptive mechanisms fail to compensate by the first three means.

\section{Signaling pathways involved in the UPR}

In mammals, the UPR includes signals initiated by ER membrane- associated proteins, such as PKR-like endoplasmic reticulum kinase (PERK), inositol requirement 1 (IRE1) and activating transcription factor (ATF)6. Activation of PERK, IRE1 $\alpha$ and ATF $6 \alpha$ in the ER is required for short-term protective response from acute stress by inhibition of translation of mRNA through eukaryotic translational initiation factor (eIF) $2 \alpha$ phosphorylation, degradation of ER-associated mRNAs by IRE1 $\alpha$, and inhibition of protein import through the translocon. ${ }^{5}$ In the absence of ER stress, GRP78 prevents PERK, IRE1 and ATF6 from being activated by binding to the lumen part of these sensors. ${ }^{1-4}$

PERK attenuates mRNA translation through phosphorylation of EIF2A

As an ER-associated type I transmembrane serine/threonine protein kinase, PERK is activated through oligomerization and transautophosphorylation. ${ }^{1-4}$ Activated PERK phosphorylates and inactivates the $\alpha$-subunit of eIF2 $\alpha$, thus inducing translational attenuation. Activation of PERK also leads to transcription of around $1 / 3$ of the UPR-dependent genes. ${ }^{1-4}$ PERK mediates mTOR, Akt, and Erk1/2 activation during ER stress. ${ }^{7}$ Importantly, PERK is required for the activation of anabolic pathways downstream of Akt in a physiological condition. ${ }^{7-8}$ Intriguingly, translation of the ATF4 is remarkably activated upon the phosphorylation of eIF $2 \alpha$ during ER stress. The targets of ATF4 include CHOP/GADD153 and GADD34, a regulatory subunit of protein phosphatase (PP)1 that targets PP1 to eIF2 $\alpha$. CHOP and GADD34 proteins are involved in amino acid biosynthesis and transport functions, antioxidative stress response, and induction of apoptosis. ${ }^{1,4}$

\section{IRE1: The conserved core of UPR}

The first stress transducer was identified by a genetic screen for mutations that suppress activation of a UPR-inducible reporter in the budding yeast Saccharomyces cerevisiae. ${ }^{6,9}$ The IRE1 $\alpha$ is a type I transmembrane protein of about $100 \mathrm{kD}$ and possesses both a serine/threonine kinase domain and a site-specific endoribonuclease domain. ${ }^{69}$ IRE1 $\alpha$ and IRE1 $\beta$ have been characterized as two mammalian homologues of yeast IRE1. The former can be detected in most cells and tissues, with highest expression levels in the pancreas and placenta. The latter is dominant only in the intestinal epithelia cells. , $^{6}$

These two isoforms share quite similar cleavage specificities, and therefore may not recognize different substrates. However, they demonstrate temporal and tissue-specific expression patterns. ${ }^{10}$ IRE $1 \alpha$ mediates a non-conventional splicing (cytoplasmic splicing) of $\mathrm{x}$-box binding protein 1 (XBP1) pre-mRNA compared with conventional mRNA splicing (nuclear splicing). ${ }^{11}$ In mammals, phosphorylated IRE1 binds the tumor necrosis factor receptor-associated factor (TRAF)2, an E3 ubiquitin ligase, to activate protein kinases involved in immunity, inflammation, and apoptosis, particularly apoptosis signal-regulating kinase 1 (ASK1)/ MAP3K5, thus activating Jun N-terminal kinase (JNK). ${ }^{4}$

The IRE1 $\alpha$-ASK1-JNK signaling pathway is also associated with a cell death mechanism due to activation of the pro-apoptotic $\mathrm{B}$ cell leukemia $(\mathrm{Bcl})-2$ family member Bcl-2-interacting mediator of cell death (BIM)/Bcl2L11 and suppression of the anti-apoptotic Bcl-2 protein. ${ }^{12-14}$ The pro-apoptotic proteins Bcl-2-associated $\mathrm{X}$ protein $(\mathrm{BAX})$ and $\mathrm{Bcl}-2$ antagonist/killer $(\mathrm{BAK})$ are found to modulate UPR signaling pathway through physical interaction with and activation of IRE $1 \alpha .^{15}$ 
ATF6 requires regulated proteolysis to mediate transcriptional activation

Like XBP1, the basic leucine zipper (bZiP)-containing ATF6 was characterized as another regulatory protein that binds to the ER stress response element 1 (ERSE1) in the promoters of UPRresponsive genes. ${ }^{16-17}$ Due to an ER-targeting hydrophobic sequence, ATF6 is tethered to ER membranes. Upon ER stress, ATF6 is released from GRP78/BiP and transports to the Golgi compartment, where it is cleaved by site 1 protease (S1P) and site 2 protease $(\mathrm{S} 2 \mathrm{P})$ resulting in a cytosolic fragment that moves to the nucleus to further activate transcription of UPR-responsive genes. ${ }^{1,16}$ Although knock-out of ATF6 $\alpha$ and ATF6 $\beta$ in the mouse, for either alone, produced no significant phenotype, combined knock-out causes early embryonic lethality. ${ }^{3}$

In contrast to the effects of ATF $6 \alpha$ on the UPR-induced genes, ATF6 $\beta$ has no regulatory function on gene expression. ${ }^{3}$ ATF $6 \alpha$ also cooperates with IRE1 to activate XBP1 expression: ATF6 $\alpha$ induces the transcription of XBP1 mRNA, which is further processed through cytoplasmic splicing by IRE1. ATF6 $\alpha$ has been shown to contain a cytoprotective activity in a variety of cellular stress models. ${ }^{18}$ Despite the fact that the specific downstream genes responsible for protecting cell survival have not been completely identified, regulator of calcineurin 1 (RCAN1) has been recognized as a potential candidate. RCAN1 is an endogenous inhibitor of calcineurin (protein phosphatase B). Calcineurin is a calcium-activated phosphatase, the substrates of which include Bcl-2 antagonist of cell death (BAD). Dephosphorylation of $\mathrm{BAD}$ by calcineurin has been reported to regain its capability to dimerize with and inhibit $\mathrm{Bcl}-\mathrm{X}_{\mathrm{L}}$, which is an anti-apoptotic protein. ${ }^{19}$

\section{GRP78/BiP is a master regulator in the UPR}

GRP78, also well known as BiP, was the first ER molecular chaperone shown to bind incompletely assembled immunoglobulin intermediates and block their transport from the ER. ${ }^{1-4,20}$ As a major ER chaperone protein with antiapoptotic properties, GRP78 is a key pro-survival component of the UPR and belongs to the heat shock protein (HSP) 70 family. ${ }^{20}$ Under rest condition, GRP78 binds to the luminal part of IRE1, PERK and ATF6. ${ }^{1-4}$ Upon ER stress, however, accumulation of misfolded or unfolded proteins in the ER lumen release GRP78 from IRE1, PERK and ATF6, activating these membrane proteins to induce the UPR or cell death. ${ }^{1-4}$ Then, GRP78 binds to the hydrophobic section of unfolded proteins through a substrate binding domain and promotes protein folding by conformational change induced by the hydrolysis of ATP through the ATPase domain. ${ }^{1-2}$

GRP78 is commonly thought to localize inside the ER lumen because of a presumed N-terminal ER localization signal that guides its localization into the ER. A highly conserved KDEL signal, common for soluble ER-localized proteins, was found at its far C-terminal end..$^{21}$ It has been reported that GRP78 can be detected in the nucleus, mitochondria, cytosol, or even the cell surface, depending on specific cell type and conditions. ${ }^{22-24}$ The discovery of GRP78 located on the surface of a different type of cells other than normal tissues suggests that there may be a specific mechanism to translocate GRP78 to the cell surface. The other possibility is that over-expression of GRP78 in the cells overloads the KDEL retrieval mechanism of ER luminal proteins, therefore inducing inadvertent transportation of GRP78 to the cell surface with client proteins. $^{25}$

\section{ER stress-induced apoptosis}

If the PERK, ATF6 and IRE1 pathways cannot help reduce or suppress ER stress for cell survival, a number of apoptotic signaling pathways are activated to ensure the organism survival as the bottom line of defense. Bcl-2 family proteins are well known factors of apoptosis machinery, and some key components participate in ER stress-induced apoptosis. ${ }^{26}$ Calcium from the ER lumen may participate in the activation of cytoplasmic proteases that promote cell death, but even how the ER stress causes the calcium leak is poorly understood. ${ }^{27}$

The CHOP pathway induced by PERK/eIF $2 \alpha$-dependent transcription activation is the most characterized pathway. ${ }^{28-30}$ The IRE1-TRAF2-ASK1-JNK pathway is the second apoptotic pathway. ${ }^{4,26,31}$ Upon ER stress, the cytoplasmic domain of IRE1 binds to TRAF2 then forms a complex with a mitogen-activated protein kinase kinase kinase (MAPKKK), also known as ASK1. This IRE1-TRAF2-ASK1 complex is in charge of the phosphorylation and activation of JNK. In addition, TRAF2 also interacts with caspase-12 and regulates its activity. ${ }^{32}$ The transcriptional repressor ATF3 can be activated by IRE1-TRAF2 and then induce activation of the apoptotic pathway, which suggests that the IRE1-TRAF2ASK1 complex may be a major regulator of ER stress-induced apoptosis. $^{33}$

During the course of ER stress, activated caspase-12 activates caspase-9, which in turn activates the downstream caspase-3, leading to apoptosis.[34] Activation of caspase-12 can be seen in many disease models, including those of Alzheimer's, polyglutamine, ischemia, and virus infection. ${ }^{35-39}$ However, functional caspase-12 can be detected only in a small subpopulation of humans because human caspase-12 has obtained a number of deleterious mutations, so that only the inactive form can be seen in most cases.[40] Therefore, the involvement of caspase-12 in apoptosis of human cells still remains to be investigated.

CHOP is the most characterized ER stress-induced apoptosis pathway

The pro-apoptotic transcription factor $\mathrm{CHOP}$ is regarded as the most significant form of ER-stress induced apoptosis. ${ }^{28-30} \mathrm{Al}-$ though other studies suggest ATF6 and XBP1 can bind to the Chop promoter region, the pathway activated through the PERK/eIF2 $\alpha$ / ATF4 branch is essential for CHOP up-regulation during chronic ER stress. ${ }^{41}$ The major molecular mechanism of CHOP's regulation of apoptosis is mainly dependent on the balance between expression levels of pro-survival $\mathrm{Bcl}-2$ proteins $\left(\mathrm{Bcl}-2, \mathrm{Bcl}-\mathrm{X}_{\mathrm{L}}\right)$ and pro-apoptotic BH3-only proteins (Bax, Bak, Bad, Bim, p53 upregulated modulator of apoptosis (PUMA) and neutrophil NADPH oxidase factor (NOXA)).

CHOP activates transcription of several genes that lead to apoptosis, including GADD $34,{ }^{42}$ death receptor 5 (DR5), ${ }^{43}$ ER oxidase (ERO1) and carbonic anhydrase VI. ${ }^{44,45}$ The GADD34-related protein phosphatase $2 \mathrm{C}$ enhances dephosphorylation of eIF $2 \alpha$ and promotes protein biosynthesis. ${ }^{41} \mathrm{Chronic}$ stress can lead to adaptation by selective attenuation of CHOP expression through degradation of Chop mRNA and CHOP protein. ${ }^{3,41}$ Accompanied by an increase in oxidative stress during chronic ER stress-induced apoptosis, the accumulation of reactive oxygen species (ROS) in the ER overcomes the various anti-oxidant response that is activated by XBP1 and PERK-Nrf2.

Further, ROS response could activate the ER calcium release channel, and then exacerbate the apoptotic signaling, including 
for FAS expression and mitochondrial calcium uptake. ${ }^{41}$ ER-mitochondrial crosstalk during ER stress leads to transfer of toxic amounts of calcium to the mitochondria, increasing the sensitivity to the mitochondrial outer membrane permeabilization (MOMP), cytochrome c release, and final induction of apoptosis. ${ }^{41}$

ER stress, Crohn's disease, intestinal inflammation and immunity

First reported in 1932 as ileitis, Crohn's disease has received a great amount of attention over the last several decades due to increasing case numbers around the globe. Now, it is considered as an immune response-mediated, chronic remittent, and relapsing inflammatory bowel disease (IBD), featuring transmural inflammation in the genetically susceptible patient. ${ }^{46,47}$ It has two major types-ulcerative colitis and Crohn's disease-with distinctive features in terms of clinical demonstration and pathogenesis. ${ }^{46,47}$ Fibrotic stricture and intestinal fibrosis can occur in about $50 \%$ of patients with Crohn's disease due to overgrowth of the muscularis propria composed of smooth muscle cell hyperplasia, hypertrophy, and excessive deposition of extracellular matrix. ${ }^{46,47}$

Inflammation serves to initiate the onset of wound healing processes in the gut, but recedes and is replaced by other domiant mechanisms contributing to fibrotic responses and final stricture formation. ${ }^{46,47}$ One mechanism responsible for early versus late colitis involves interleukin (IL)-12 playing a pivotal role in the early stage, while IL-4 and IL-13 sustain chronic inflammation in the late disease, as shown in an IL-10-deficient colitis mouse model and indicating that distinct inflammatory mechanisms may control different stages of colonic inflammation. ${ }^{48}$

The other potential mechanism of fibrosis is that mesenchymal cells participate in a later stage of fibrogenesis involved in stricture formation. ${ }^{46,47}$ Imbalance between tissue inhibitor of matrix metalloproteinases (TIMPs) and matrix metalloproteinases (MMPs) partially contribute to the development of intestinal fibrosis in patients with Crohn's disease as well as in animal models of liver fibrosis. ${ }^{46,47}$ Using a transgenic mouse over-expressing human (h) TIMP-1 in the liver, Youshiji et al. ${ }^{49}$ showed that TIMP-1 does not result in liver fibrosis by itself, but strongly promotes liver fibrosis development. Murphy et al..$^{50}$ demonstrated in experimental liver cirrhosis that persistent expression of TIMP-1 mRNA with persistence of activated $\alpha$-smooth muscle actin (SMA)-staining hepatic stellate cells (HSCs) in fibrosis have loss of activated HSCs, which is correlated with a reduction in TIMP-1 mRNA. Taken together, these data indicate that the MMP and TIMP system may play a role in the sustaining of fibrosis during ER stress but not as an initiator, while TIMP-1 inhibits apoptosis of activated HSC via MMP inhibition.

ER stress and immunity are usually intertwined together during the different stages of the inflammatory process in a variety of human diseases. ${ }^{51,52}$ This complexity of interaction has been further evidenced using knock-out animals. ${ }^{53-56}$ In $\mathrm{CHOP}^{-/-}$mice, bleomycin-induced lung fibrosis was significantly attenuated compared to that in wild-type mice. Administration of tauroursodeoxycholic acid, a chemical chaperone, inhibited bleomycin-induced inflammation and fibrosis in mice. ${ }^{53}$ In Endo et al.'s report, ${ }^{54}$ lipopolysaccharide-induced inflammation in lung of $\mathrm{CHOP}^{-1-}$ mice was also attenuated, as was neutrophil infiltration and IL-1 $\beta$ and caspase-11 expression. However, in contrast to those studies, Ayaub et $a l .{ }^{55}$ showed that quasi-static elastance (also known as the elastic resistance that is the reciprocal of compliance) and extracellular matrix $(\mathrm{ECM})$ deposition were increased with proliferation of argi- nase-1-positive lung macrophages in $\mathrm{CHOP}^{-/-}$mice. Paradoxically, GRP78 ${ }^{+/-}$haploinsufficiency mice were significantly protected against bleomycin-induced lung fibrosis due to a reduced number of lung macrophages with positivity for cleaved caspase- $3 .{ }^{55}$

These data collectively suggest that GRP78- and CHOP-mediated macrophage apoptosis may have opposite roles in response to bleomycin-induced fibrosis and also indicate the potential role of macrophages in this animal model of fibrosis. More interestingly, in a mouse model of nonalcoholic steatohepatitis, $\mathrm{CHOP}^{-/-}$mice demonstrated greater liver damage, inflammation, and fibrosis compared to $\mathrm{CHOP}$ wild-type $\left(\mathrm{CHOP}^{+/+}\right)$, due to sustained survival of activated macrophages. Persistence of net accumulation of these activated macrophages in the liver potentiates liver steatohepatitis in $\mathrm{CHOP}^{-/-}$mice. ${ }^{56}$ Yao et al. ${ }^{57}$ reported that $\mathrm{CHOP}$ deficiency inhibited the alternatively activated macrophage phenotype (M2) and reduced its filtration in the mouse lung after bleomycin induction. These activated M2 macrophages can produce transforming growth factor (TGF)- $\beta$ and platelet-derived growth factor to induce myofibroblast activation and trigger tissue fibrosis.

Taken together, the role of each individual mediator during the ER stress response including CHOP and GRP78 should be examined and interpreted with caution and according to the different experimental settings. Each may have opposite effects on distinct cell types, tissues and disease context, as well as on macrophage activation and proliferation.

ER stress can also be blocked with anti-inflammatory treatment. In primary intestinal epithelial cells of inflamed IL $10^{-/-}$mice and IBD patients, activated ER stress response was associated with increased GRP78 under chronic inflammation, which can be completely blocked with Grp78 knock-down or by adding IL-10 to tumor necrosis factor (TNF)-stimulated IL-10 receptor-overexpressing epithelial cells. ${ }^{58}$ The anti-ER stress effect of IL-10 was due to IL-10-induced p38 activation and blockage of nuclear translocation and recruitment of ATF6 to the Grp 78 promoter. ${ }^{58}$ The cited study suggests that dysregulation of ER stress in the absence of anti-inflammatory cytokine in epithelium may contribute to chronic inflammation-induced intestinal epithelial damage.

It has become clear that ER stress signaling plays an important role in immune response and inflammation during the pathogenesis of fibrosis as well as in many other autoimmune diseases. ${ }^{51,52}$ During chronic/adaptive ER stress, the UPR induces a series of cellular events to maintain a proper protein homeostasis and therefore restore the regular cellular function including glycosylation for protein folding, oxidative stress, calcium translocation, and autophagy. These same principles apply to the immune cells through cellular differentiation and the appropriate immune response in the mucosal epithelial. ${ }^{51,52}$ Activation of components of the innate and adaptive immune systems plays an important role in the development of chronic intestinal inflammation and therefore lead to overactive wound healing processes and activation of myofibroblasts. ${ }^{51,52}$

Early reports showed that loss of XBP1 in the intestinal epithelial cells of $\mathrm{XBP}^{-/-}$mice caused progressive Paneth cell death and spontaneous inflammation in the mouse ileum. ${ }^{59}$ Although loss of eIF $2 \alpha$ phosphorylation did not affect the normal intestinal epithelial cell proliferation or differentiation in $A A^{I E C}$ mice (which expressed non-phosphorylatable Ser51Ala mutant eIF2 $\alpha$ in the intestinal epithelial cells), the UPR gene expression was defective in these mice; these mice were also more susceptible to dextran sulfate sodium-induced colitis. ${ }^{60} \mathrm{~A}$ similar pattern of severe inflammation was also found in the knock-out mice of ATF $6 \alpha$ and the protein chaperone $\mathrm{p} 58^{\mathrm{IPK}}$ as well as in IL- $10^{-/-}$mice. ${ }^{61}$ Taken together, these data showed the complex interplay between ER stress, inflammation and immunity.

However, there are still many unanswered questions that await 
further investigation. For example, how these different T cells mediate immune response according to specific environmental ER stressors? How different cytokines coordinate a robust cellular signaling network to contribute to intestinal fibrosis? How specific immunological modulation in different type of immune cells affects the development of intestinal fibrosis? These questions need to be addressed systematically in studies using cells and animals that are deficient in specific ER stress sensors, particularly in specific cell types, when challenged with different exogenous environmental conditions.

Treatment targeting single cytokines without a full understanding of the changes in immune response in the whole-organism context are primed for failure, as shown by the failure of anti-IL-17A in Crohn's disease. ${ }^{52}$ Moreover, in a study of the lung inflammatory and fibrotic responses to silica of MyD88 knock-out mice, robust collagen accumulation was observed in knock-out mice in spite of attenuation of lung inflammation, neutrophil accumulation and IL- $1 \beta$ release in response to silica. ${ }^{62}$ In that same study, fibrosis was associated with increased levels of pro-fibrotic regulatory $\mathrm{T}$ lymphocytes but not with $\mathrm{T}$ helper 17 cell influx. ${ }^{62}$ The data suggest that the development of lung fibrosis or even other fibrotic diseases appears uncoupled from inflammation and may be orchestrated by a regulatory T lymphocyte-associated pathway. ${ }^{62}$

Several ER modulating therapeutic interventions showed that treatment with chemical chaperones improves protein folding in disorders of misfolded proteins, such as alpha-1 antitrypsin deficiency. ${ }^{63}$ Agents that ameliorate ER stress by promoting adaptive UPR signaling and/or preventing ER stress-mediated cell apoptosis offer a promising therapy target, such as CHOP inhibitor to reduce inflammation-induced epithelial cell damage. ${ }^{57,64}$ Concern should be also raised when this same agent is applied to mesenchymal cells, because it may exacerbate the development of fibrosis through activation of myofibroblasts by TGF- $\beta$ secretion from activated macrophages. ${ }^{56}$

In our study, decreased levels of apoptosis proteins and Chop mRNA were found in affected ileum of patients with fibrostenotic Crohn's disease, as compared to the normal margin in the same patient as well as other phenotypes. ER stress response with the feature of GRP78, XBP1s and ATF6 $\alpha$ over-expression can be also activated in the normal mesenchymal cells when treated with tunicamycin. ${ }^{64,65}$ The increased amount of ER stress in affected ileum was found to be associated with increased silencing of microRNA (miR)-199a-5p by DNMT1-mediated promoter hypermethylation. ${ }^{64,65}$ In the at-rest condition, miR-199a-5p targets different ER stressors including GRP78, ATF6 and XBP1s for their degradation $^{64,65}$ (Fig. 1). Restoration of miR-199a-5p through a DNA methylation inhibitor, such as 5-azacytidine, to inhibit DNMT1 function may suppress activated ER stress-induced myofibroblast activation and excess ECM production, and finally prevent intestinal fibrosis (Fig. 1). ${ }^{64,65}$

\section{ER stress, autophagy and inflammation: crosstalk}

The crosstalk between ER stress, autophagy, and inflammation has received gradual attention for its role in inflammatory processes in IBD. Unresolved and deregulated ER stress is a common feature in inflamed epithelium of the gut and affects key functions of the epithelial barrier, which might shed light on the mechanism of susceptibility of mucosa to environmental stress. ${ }^{66}$ The UPR and autophagy are intertwined signaling pathways that can compensate for the loss of each other in the intestinal epithelium. ${ }^{67}$ Adolph et al ${ }^{67}$ showed that $X b p 1^{4 I E C}$ mice demonstrated autophagosome formation in hypomorphic Paneth cells, which is associated with ER stress via PERK, eIF2 $\alpha$ and ATF4, indicating a compensatory effect from one of the three-pronged UPR pathways (the other two are IRE1 $\alpha$-XBP1 and ATF6) to promote autophagy. Moreover, in Atg $1611^{\triangle I E C}$ mice with deficient autophagy in intestinal epithelium, intestinal epithelial cell death, IRE1 $\alpha$-regulated NF- $\kappa$ B activation and TNF signaling were synergistically enhanced. ER stress, autophagy and spontaneous ileitis take place upon Paneth cell-specific deletion of $X b p 1$. Collectively, these data suggest the complicated relationship between UPR and autophagy existing in intestinal epithelium is necessary to maintain intestinal homeostasis.

However, autophagy activated by ER stress inducers can alleviate UPR and reduce cell death in cancer cells but not in nontransformed cells, which suggest the differential role of autophagy that is played according to the different status of cells. ${ }^{68}$ Lopes et $a l .{ }^{69}$ reported that ATF6 enhanced autophagic killing of bacteria, thereby preventing damage of epithelial barrier function that was caused by dysfunctional mitochondria. Promotion of autophagy amid ER stress protects further intestinal damage. Whether ER stress is a consequence or mechanism to compensate for defective intracellular function, the ultimate outcome would be more worthy of consideration. For example, does it lead to more severe damage in the gut or protect it from further attack through activation of autophagy? This kind of question should be addressed in a more specific cellular system. In our view, whether induction of autophagy by ER stress in mesenchymal cells coordinately maintains colonic intracellular homeostasis or inappropriately contributes to intestinal fibrosis requires further elegant study.

\section{Future directions}

ER stress plays a critical role in different types of cells implicated in the pathogenesis of IBD and intestinal fibrosis. The outcome is distinctive and cell context-dependent as discussed here in intestinal epithelial and mesenchymal cells. Even though more than 200 genetic loci associated with IBD have been uncovered, new targets and their functional roles in IBD pathophysiology still remain unknown. Moreover, the environment, the genome, and the microbiota have also been well recognized and integrated into our understanding of pathogenesis of IBD. ${ }^{70}$ How to integrate these findings into our understanding of intracellular organelle disorders such as ER stress lags far behind. Based on numerous studies from the past several decades, we realize fibrosis occurs in all forms of IBD and non-IBD intestinal inflammation. Fibrosis is reversible and curable, for it may not be the permanent status of disease. In addition to the traditional anti-inflammatory regimen, we need to consider the following options in terms of druggable treatments: ${ }^{70}$

1. Targeting critical ER components to reduce intracellular protein overload and restore intestinal homeostasis;

2. Targeting ECM and reducing collagen deposition to lessen the stricture formation;

3. Targeting mesenchymal cell differentiation, dedifferentiation, and tissue remodeling to prevent myofibroblast proliferation.

Restoration of intracellular ER homeostasis leads to several important mechanistic questions: Is ER stress the cause, the result, or both of intestinal inflammation? Can ER stress occur in the absence of inflammation? Which UPR pathway(s) is(are) critical in the pathogenesis of intestinal fibrosis? How do they activate or inactivate apoptosis in the different types of cells in the gut? What are the relationships between intestinal epithelia and muscular tissue (smooth muscle cell) and/or any other type of cells inside the 


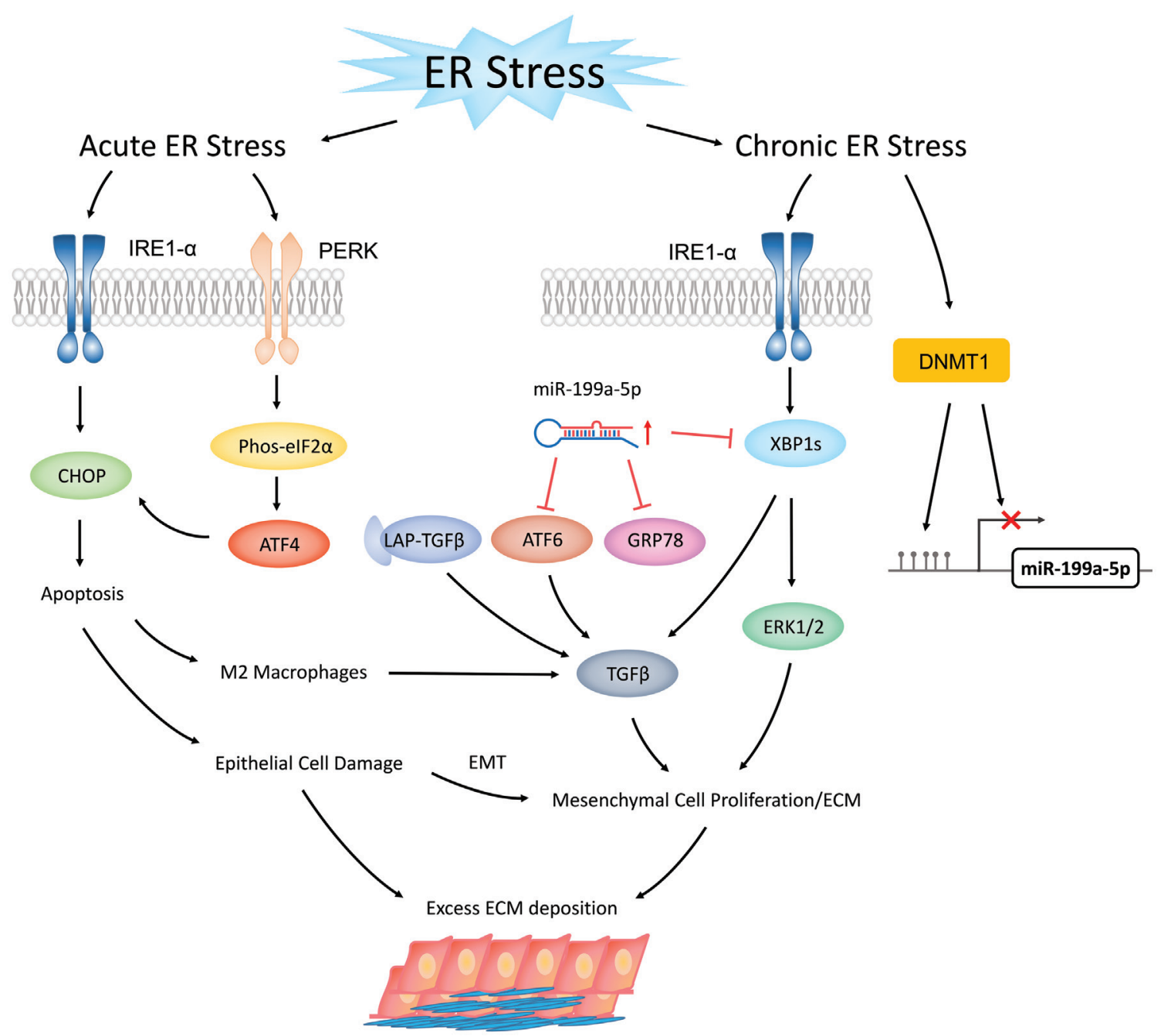

Fig. 1. The hypothesis of dual roles of ER stress and UPR in the development of intestinal fibrosis in Crohn's disease. When intestinal epithelial cells (IECS) are subject to acute ER stress, ER stress sensors, such as IRE1 $\alpha$ and PERK, can be activated by detached association with GRP78. Individual downstream signaling, including that of CHOP and elF2 $\alpha /$ ATF4, are further activated to induce apoptosis of IECs. After CHOP induces activation of macrophages (M2), TGF- $\beta$ can be secreted from M2 to activate mesenchymal cells in the gut to induce ECM protein production. When the intestine becomes adaptive to chronic inflammation-induced ER stress, the IRE1 $\alpha$ /XBP1 ER stress branch activates ERK1/2 to stimulate mesenchymal cell proliferation. The increased UPR is also associated with increased silencing of miR-199a-5p by DNMT1-mediated promoter hypermethylation. In the at-rest condition, miR-199a-5p targets different ER stressors including GRP78, ATF6, and XBP1s for their degradation. ATF6 and XBP1 activate ER stress-induced myofibroblast activation through up-regulation of TGF- $\beta$ and excess ECM production. GRP78 can bind to latent associated peptide-TGF- $\beta$ to activate TGF- $\beta$. All these factors can finally lead to intestinal fibrosis. Refer to context for details. Abbreviations: ATF, activating transcription factor; CHOP, C/EBP homologous protein; ECM, extracellular matrix; EMT, Epithelial-mesenchymal transition; ER, endoplasmic reticulum; elF, eukaryotic translational initiation factor; GRP, glucose-regulated protein; IRE1, inositol requirement 1; PERK, PKR-like endoplasmic reticulum kinase; TGF, transforming growth factor; UPR, unfolded protein response; XBP1, $x$-box binding protein 1.

colon (i.e. immune cells including macrophages, T cells, pericytes, Paneth cells, myofibroblasts and fibroblasts).

ER stress is also reported to be associated with epithelial-mesenchymal transition in alveolar epithelial cells. ${ }^{71}$ ER stress inducers, thapsigargin or tunicamycin, increased expression of Grp78 and XBP1s, decreased epithelial markers E-cadherin and zonula occludens-1 (ZO-1), increased $\alpha$-SMA, and induced fibroblast-like morphology in primary epithelial cells, consistent with epithelialmesenchymal transition. ${ }^{71}$ Enhanced proliferation of collagen-secreting mesenchymal cells and defective programmed cell death of those cells are considered to implicate in excessive ECM deposition in the gut. ${ }^{64,71}$

Occurrence of "modern diseases" is related to Western diet, environmental factors, psychological issues and life style. ${ }^{72}$ Interestingly, changing the diet from a methionine choline-deficient diet to a methionine choline control diet led to resolution of hepatic inflammatory and fibrotic reactions and hepatocyte apoptosis through inhibition of ER stress, as shown in methionine cholinedeficient diet-induced steatohepatitis mice. ${ }^{73}$ That study suggests that early intervention with healthy diet and life style may still have the potential to reverse fibrosis in the early stage. ${ }^{73}$

\section{Conclusions}

ER stress and UPR represent a conserved signaling network pre- 
sent in various organisms, from yeast to mammals. The UPR is activated in both acute and chronic ER stress, with corresponding cellular adaption. Apoptosis is usually activated to clear the damaged cells when they are not able to process the disturbance of intestinal homeostasis due to intracellular accumulation of misfolded proteins or large amount of regular proteins. Intestinal fibrosis is a multifactorial process, and a paradigm shift of the anti-fibrotic treatment relies on our fundamental understanding of the molecular mechanisms of fibrosis. ER stress plays a major role in intestinal fibrosis depending on the specific cell type in the gut. The use of intestinal-mesenchymal cell type in a mouse model with knockout or knock-in of critical components of the UPR may facilitate the development of a novel antifibrotic regimen.

As illustrated in this review, ER stress plays a dual role in the development of intestinal fibrosis, as featured by its induction of apoptosis in intestinal epithelial cells on the one side and its promoting of exaggerated adaptive, survival-associated UPR signaling in mesenchymal cell cells on the other side. Thus, it is unequivocal to conjecture that the general outcome of this dysregulated UPR in Crohn's disease might be dependent on manipulation of cell population balance between these two distinct cell types under adaptive ER stress. Therapeutic strategies to restore ER homeostasis may be essential for the treatment of intestinal fibrosis. Yet, concern should be taken to evaluate the potential pitfall of whether systemic suppression of ER stress is beneficial for patients with a specific phenotype, such as inflammatory versus fibrostenotic. Selective inhibition of ER stress in specific cell types, such as mesenchymal cells, to prevent cell proliferation with excess collagen deposition and in epithelial cells to skip apoptosis-induced mucosal damage might lead to a tailored individual therapy approach in the future.

\section{Acknowledgments}

I apologize to all colleagues whose work could not be cited due to space limitations. This work has been funded by grants from the Virginia Commonwealth University (VCU)'s CTSA (UL1TR000058 from the National Center for Advancing Translational Sciences) \& the CCTR Endowment Fund of VCU and from the Crohn's \& Colitis Foundation (Ref. \#550514).

\section{Conflict of interest}

The author declares no conflict of interest with this publication.

\section{Author contributions}

Conceiving of the presented idea, performance of the literature research, collection and analysis of the references, and writing of the manuscript (CL).

\section{References}

[1] Yoshida H. ER stress and diseases. FEBS J 2007;274(3):630-658. doi:10.1111/j.1742-4658.2007.05639.x.

[2] Rutkowski DT, Kaufman RJ. That which does not kill me makes me stronger: adapting to chronic stress. Trends Biochem Sci 2007;32(10):469-476. doi:10.1016/j.tibs.2007.09.003.

[3] Malhotra JD, Kaufman RJ. The endoplasmic reticulum and the un- folded protein response. Semi in Cell \& Dev Biol 2007;18(6):716-731. doi:10.1016/j.semcdb.2007.09.003.

[4] Ron D, Walter P. Signal integration in the endoplasmic reticulum un folded protein response. Nat Rev Mol Cell Biol 2007;8(7):519-529. doi:10.1038/nrm2199.

[5] Kim I, Xu W, Reed JC. Cell death and endoplasmic reticulum stress: disease relevance and therapeutic opportunities. Nat Rev Drug Discov 2008;7(12):1013-1030. doi:10.1038/nrd2755.

[6] Tirasophon W, Welihinda AA, Kaufman RJ. A stress response pathway from the endoplasmic reticulum to the nucleus requires a novel bifunctional protein kinase/endoribonuclease (Ire1p) in mammalian cells. Genes Dev 1998;12(12):1812-1824. doi:10.1101/ gad.12.12.1812.

[7] Bobrovnikova-Marjon E, Pytel D, Riese MJ, Vaites LP, Singh N, Koretzky GA, et al. PERK utilizes intrinsic lipid kinase activity to generate phosphatidic acid, mediate Akt activation, and promote adipocyte differentiation. Mol Cell Biol 2012;32(12):2268-2278. doi:10.1128/ MCB.00063-12.

[8] Darling NJ, Balmanno K, Cook SJ. ERK1/2 signalling protects against apoptosis following endoplasmic reticulum stress but cannot provide long-term protection against BAX/BAK-independent cell death. PLoS One 2017;12(9):e0184907. doi:10.1371/journal.pone.0184907.

[9] Wang XZ, Harding HP, Zhang Y, Jolicoeur EM, Kuroda M, Ron D. Cloning of mammalian Ire1 reveals diversity in the ER stress responses. EMBO J 1998;17(19):5708-5717. doi:10.1093/emboj/17.19.5708.

[10] Niwa M, Sidrauski C, Kaufman RJ, Walter P. A role for presenilin-1 in nuclear accumulation of Ire1 fragments and induction of the mammalian unfolded protein response. Cell 1999;99(7):691-702. doi:10.1016/S0092-8674(00)81667-0.

[11] Yoshida H, Matsui T, Yamamoto A, Okada T, Mori K. XBP1 mRNA is induced by ATF6 and spliced by IRE1 in response to ER stress to produce a highly active transcription factor. Cell 2001;107(7):881-891. doi:10.1016/S0092-8674(01)00611-0.

[12] Lei K, Davis RJ. JNK phosphorylation of Bim-related members of the Bcl2 family induces Bax-dependent apoptosis. Proc Natl Acad Sci U S A 2003;100(5):2432-2437. doi:10.1073/pnas.0438011100.

[13] Putcha GV, Le S, Frank S, Besirli CG, Clark K, Chu B, et al. JNK-mediated BIM phosphorylation potentiates BAX-dependent apoptosis. Neuron 2003;38(6):899-914. doi:10.1016/S0896-6273(03)00355-6.

[14] Yamamoto K, Ichijo H, Korsmeyer SJ. BCL-2 is phosphorylated and inactivated by an ASK1/Jun N-terminal protein kinase pathway normally activated at G2/M. Mol Cell Biol 1999;19(12):8469-8478. doi:10.1128/MCB.19.12.8469.

[15] Hetz C, Bernasconi P, Fisher J, Lee AH, Bassik MC, Antonsson B, et al. Proapoptotic BAX and BAK modulate the unfolded protein response by a direct interaction with IRE1 $\alpha$. Science 2006;312(5773):572-576 doi:10.1126/science. 1123480 .

[16] Okada T, Yoshida H, Akazawa R, Negishi M, Mori K. Distinct roles of activating transcription factor 6 (ATF6) and double-stranded RNA activated protein kinase-like endoplasmic reticulum kinase (PERK) in transcription during the mammalian unfolded protein response. Biochem J 2002;366(Pt 2):585-594. doi:10.1042/BJ20020391.

[17] Yoshida H, Haze K, Yanagi H, Yura T, Mori K. Identification of the cisacting endoplasmic reticulum stress response element responsible for transcriptional induction of mammalian glucose-regulated proteins. Involvement of basic leucine zipper transcription factors. J Biol Chem 1998;273(50):33741-33749. doi:10.1074/jbc.273.50.33741.

[18] Thuerauf DJ, Marcinko M, Belmont PJ, Glembotski CC. Effects of the isoform-specific characteristics of ATF6 $\alpha$ and ATF6 $\beta$ on endoplasmic reticulum stress response gene expression and cell viability. J Biol Chem 2007;282(31):22865-22878. doi:10.1074/jbc.M701213200.

[19] Wang HG, Pathan N, Ethell IM, Krajewski S, Yamaguchi Y, Shibasaki $\mathrm{F}$, et al. Ca2+-induced apoptosis through calcineurin dephosphorylation of BAD. Science 1999;284(5412):339-343. doi:10.1126/science.284.5412.339.

[20] Haas IG, Wabl M. Immunoglobulin heavy chain binding protein. Nature 1983;306(5941):387-389. doi:10.1038/306387a0.

[21] Daugaard $M$, Rohde $M$, Jäättelä $M$. The heat shock protein 70 family: highly homologous proteins with overlapping and distinct functions. FEBS Lett 2007;581(19):3702-3710. doi:10.1016/j.febslet.2007.05.039. 
[22] Zhang Y, Liu R, Ni M, Gill P, Lee AS. Cell surface relocalization of the endoplasmic reticulum chaperone and unfolded protein response regulator GRP78/BiP. J Biol Chem 2010;285(20):15065-15075. doi:10.1074/jbc.M109.087445.

[23] Shin BK, Wang H, Yim AM, Le Naour F, Brichory F, Jang JH, et al. Global profiling of the cell surface proteome of cancer cells uncovers an abundance of proteins with chaperone function. J Biol Chem 2003;278(9):7607-7616. doi:10.1074/jbc.M210455200.

[24] Misra UK, Deedwania R, Pizzo SV. Activation and cross-talk between Akt, NF-Kb, and unfolded protein response signaling in 1-LN prostate cancer cells consequent to ligation of cell surface-associated GRP78. J Biol Chem 2006;281(19):13694-13707. doi:10.1074/jbc. M511694200.

[25] Li J, Lee AS. Stress induction of GRP78/BiP and its role in cancer. Curr Mol Med 2006;6(1):45-54. doi:10.2174/156652406775574523.

[26] Heath-Engel HM, Chang NC, Shore GC. The endoplasmic reticulum in apoptosis and autophagy: role of the $\mathrm{BCL}-2$ protein family. Oncogene 2008;27(50):6419-6433. doi:10.1038/onc.2008.309.

[27] Nakagawa T, Yuan J. Cross-talk between two cysteine protease families. Activation of capspase-12 by calpain in apoptosis. J Cell Biol 2000;150(4):887-894. doi:10.1083/jcb.150.4.887.

[28] Yoshida H, Matsui T, Yamamoto A, Okada T, Mori K. XBP1 mRNA is induced by ATF6 and spliced by IRE1 in response to ER stress to produce a highly active transcription factor. Cell 2001;107(7):881-891. doi:10.1016/S0092-8674(01)00611-0.

[29] Ma Y, Brewer JW, Diehl JA, Hendershot LM. Two distinct stress signaling pathways converge upon the CHOP promoter during the mammalian unfolded protein response. J Mol Biol 2002;318(5):13511365. doi:10.1016/S0022-2836(02)00234-6.

[30] Ron D, Habener JF. CHOP, a novel developmentally regulated nuclear protein that dimerizes with transcription factors C/EBP and LAP and functions as a dominant- negative inhibitor of gene transcription. Genes Dev 1992;6(3):439-453. doi:10.1101/gad.6.3.439.

[31] Urano F, Wang X, Bertolotti A, Zhang Y, Chung P, Harding HP, et al. Coupling of stress in the ER to activation of JNK protein kinases by transmembrane protein kinase IRE1. Science 2000;287(5453):664666. doi:10.1126/science.287.5453.664.

[32] Yoneda T, Imaizumi K, Oono K, Yui D, Gomi F, Katayama T, et al. Activation of caspase-12, an endoplastic reticulum (ER) resident caspase, through tumor necrosis factor receptor-associated factor 2-dependent mechanism in response to the ER stress. J Biol Chem 2001;276(17):13935-13940. doi:10.1074/jbc.M010677200.

[33] Zhang C, Kawauchi J, Adachi MT, Hashimoto Y, Oshiro S, Aso T, et al. Activation of JNK and transcriptional repressor ATF3/LRF1 through the IRE1/TRAF2 pathway is implicated in human vascular endothelial cell death by homocysteine. Biochem Biophys Res Commun 2001;289(3):718-724. doi:10.1006/bbrc.2001.6044.

[35] Bitko V, Barik S. An endoplasmic reticulumspecific stress-activated caspase (caspase-12) is implicated in the apoptosis of A549 epithelial cells by respiratory syncytial virus. J Cell Biochem 2001;80(3):441-454. doi:10.1002/1097-4644(20010301)80:3<441::AID-JCB170>3.0.CO;2C.

[36] Kouroku Y, Fujita E, Jimbo A, Kikuchi T, Yamagata T, Momoi MY, et al. Polyglutamine aggregates stimulate ER stress signals and caspase-12 activation. Hum Mol Genet 2002;11(13):1505-1515. doi:10.1093/ hmg/11.13.1505.

[37] Mouw G, Zechel JL, Gamboa J, Lust WD, Selman WR, Ratcheson RA. Activation of caspase-12, an endoplasmic reticulum resident caspase, after permanent focal ischemia in rat. Neuroreport 2003;14(2):183186. doi:10.1097/01.wnr.0000054277.81804.24.

[38] Nakagawa T, Zhu H, Morishima N, Li E, Xu J, Yankner BA, et al. Caspase-12 mediates endoplasmic-reticulum-specific apoptosis and cytotoxicity by amyloid-beta. Nature 2000;403(6765):98-103. doi:10.1038/47513.

[39] Shibata M, Hattori H, Sasaki T, Gotoh J, Hamada J, Fukuuchi Y. Activation of caspase- 12 by endoplasmic reticulum stress induced by transient middle cerebral artery occlusion in mice. Neuroscience 2003;118(2):491-499. doi:10.1016/S0306-4522(02)00910-7.

[41] Verfaillie T, Jager R, Samali A, Agostinis P. ER stress signaling pathways in cell survival and death. In: Agostinis P, Afshin S., editors. Endoplasmic Reticulum Stress in Health and Disease. Springer, Netherlands.
2012, pp. 41-73.

[42] Novoa I, Zeng H, Harding HP, Ron D. Feedback inhibition of the unfolded protein response by GADD34-mediated dephosphorylation of elF2alpha. J Cell Biol 2001;153(5):1011-1022. doi:10.1083/ jcb.153.5.1011.

[43] Yamaguchi H, Wang HG. CHOP is involved in endoplasmic reticulum stress-induced apoptosis by enhancing DR5 expression in human carcinoma cells. J Biol Chem 2004;279(44):45495-45502. doi:10.1074/ jbc.M406933200

[44] Marciniak SJ, Yun CY, Oyadomari S, Novoa I, Zhang Y, Jungreis R, et al. $\mathrm{CHOP}$ induces death by promoting protein synthesis and oxidation in the stressed endoplasmic reticulum. Genes Dev 2004;18(24):30663077. doi:10.1101/gad.1250704.

[45] Sok J, Wang XZ, Batchvarova N, Kuroda M, Harding H, Ron D. CHOPDependent stress-inducible expression of a novel form of carbonic anhydrase VI. Mol Cell Biol 1999;19(1):495-504. doi:10.1128/ MCB.19.1.495.

[46] Li C, Kuemmerle JF. The mechanisms that mediate the development of fibrosis in patients with Crohn's disease. Inflamm Bowel Dis 2014;20(7):1250-1258. doi:10.1097/MIB.0000000000000043.

[47] Rieder F, Fiocchi C, Rogler G. Mechanisms, management, and treatment of fibrosis in patients with inflammatory bowel diseases. Gastroenterology 2017;152(2):340-350.e6. doi:10.1053/j.gastro.2016.09.047.

[48] Keubler LM, Buettner M, Häger C, Bleich A. A multihit model: colitis lessons from the interleukin-10-deficient mouse. Inflamm Bowel Dis 2015;21(8):1967-1975. doi:10.1097/MIB.0000000000000468.

[49] Yoshiji H, Kuriyama S, Miyamoto Y, Thorgeirsson UP, Gomez DE, Kawata $\mathrm{M}$, et al. Tissue inhibitor of metalloproteinases-1 promotes liver fibrosis development in a transgenic mouse model. Hepatology 2000;32(6):1248-1254. doi:10.1053/jhep.2000.20521.

[50] Murphy FR, Issa R, Zhou X, Ratnarajah S, Nagase H, Arthur MJ, et al. Inhibition of apoptosis of activated hepatic stellate cells by tissue inhibitor of metalloproteinase- 1 is mediated via effects on matrix metalloproteinase inhibition: implications for reversibility of liver fibrosis. J Biol Chem 2002;277(13):11069-11076.

[51] Bettigole SE, Glimcher LH. Endoplasmic reticulum stress in immunity. Annu Rev Immunol 2015;33:107-138. doi:10.1146/annurev-immunol-032414-112116.

[52] Geremia A, Biancheri P, Allan P, Corazza GR, Di Sabatino A. Innate and adaptive immunity in inflammatory bowel disease. Autoimmun Rev 2014;13(1):3-10. doi:10.1016/j.autrev.2013.06.004.

[53] Tanaka Y, Ishitsuka Y, Hayasaka M, Yamada Y, Miyata K, Endo M, et al. The exacerbating roles of CCAAT/enhancer-binding protein homologous protein (CHOP) in the development of bleomycin-induced pulmonary fibrosis and the preventive effects of tauroursodeoxycholic acid (TUDCA) against pulmonary fibrosis in mice. Pharmacol Res 2015;99:52-62. doi:10.1016/j.phrs.2015.05.004.

[54] Endo M, Mori M, Akira S, Gotoh T. C/EBP homologous protein (CHOP) is crucial for the induction of caspase-11 and the pathogenesis of lipopolysaccharide-induced inflammation. J Immunol 2006;176(10):6245-6253. doi:10.4049/jimmunol.176.10.6245.

[55] Ayaub EA, Kolb PS, Mohammed-Ali Z, Tat V, Murphy J, Bellaye PS, et al. GRP78 and CHOP modulate macrophage apoptosis and the development of bleomycin-induced pulmonary fibrosis. J Pathol 2016;239(4):411-425. doi:10.1002/path.4738.

[56] Malhi H, Kropp EM, Clavo VF, Kobrossi CR, Han J, Mauer AS, et al. C/EBP homologous protein-induced macrophage apoptosis protects mice from steatohepatitis. J Biol Chem 2013;288(26):18624-18642 doi:10.1074/jbc.M112.442954.

[57] Yao Y, Wang Y, Zhang Z, He L, Zhu J, Zhang M, et al. Chop deficiency protects mice against bleomycin-induced pulmonary fibrosis by attenuating M2 macrophage production. Mol Ther 2016;24(5):915925. doi:10.1038/mt.2016.36.

[58] Shkoda A, Ruiz PA, Daniel H, Kim SC, Rogler G, Sartor RB, et al. Interleukin-10 blocked endoplasmic reticulum stress in intestinal epithelial cells: impact on chronic inflammation. Gastroenterology 2007;132(1):190-207. doi:10.1053/j.gastro.2006.10.030.

[59] Kaser A, Lee AH, Franke A, Glickman JN, Zeissig S, Tilg $\mathrm{H}$, et al. XBP1 links ER stress to intestinal inflammation and confers genetic risk for human inflammatory bowel disease. Cell 2008;134(5):743-756. 
doi:10.1016/j.cell.2008.07.021.

[60] Cao SS, Wang M, Harrington JC, Chuang BM, Eckmann L, Kaufman RJ. Phosphorylation of elF2 $\alpha$ is dispensable for differentiation but required at a posttranscriptional level for paneth cell function and intestinal homeostasis in mice. Inflamm Bowel Dis 2014;20(4):712722. doi:10.1097/MIB.0000000000000010.

[61] Cao SS, Zimmermann EM, Chuang BM, Song B, Nwokoye A, Wilkinson JE, et al. The unfolded protein response and chemical chaperones reduce protein misfolding and colitis in mice. Gastroenterology 2013;144(5):989-1000.e6. doi:10.1053/j.gastro.2013.01.023.

[62] Re SL, Giordano G, Yakoub Y, Devosse R, Uwambayinema F, Couillin I, et al. Uncoupling between inflammatory and fibrotic responses to silica: evidence from MyD88 knockout mice. PLoS One 2014;9(7):e99383. doi:10.1371/journal.pone.0099383.

[63] Kelly E, Greene CM, Carroll TP, McElvaney NG, O’Neill SJ. Selenoprotein S/SEPS1 modifies endoplasmic reticulum stress in $Z$ variant alpha1-antitrypsin deficiency. J Biol Chem 2009;284(25):16891-16897. doi:10.1074/jbc.M109.006288.

[64] Li C, Kuemmerle JF. 320 ER stress in subepithelial myofibroblasts increases the TGF- $\beta 1$ activity that regulates fibrosis in Crohn's disease. Gastroenterology 2016;150(4):S75. doi:10.1016/S00165085(16)30366-3.

[65] Li C, Kuemmerle JF. Increased ER stress and unfolded protein response in subepithelial myofibroblasts contributes to fibrosis in Crohn's disease. Inflamm Bowel Dis 2016(suppl 1):S56. doi:10.1097/01. MIB.0000480248.94772.21.

[66] Tréton X, Pédruzzi E, Cazals-Hatem D, Grodet A, Panis Y, Groyer A, et al. Altered endoplasmic reticulum stress affects translation in inac- tive colon tissue from patients with ulcerative colitis. Gastroenterology 2011;141(3):1024-1035. doi:10.1053/j.gastro.2011.05.033.

[67] Adolph TE, Tomczak MF, Niederreiter L, Ko HJ, Böck J, Martinez-Naves $\mathrm{E}$, et al. Paneth cells as a site of origin for intestinal inflammation. Nature 2013;503(7475):272-276. doi:10.1038/nature12599.

[68] Ding WX, Ni HM, Gao W, Hou YF, Melan MA, Chen X, et al. Differential effects of endoplasmic reticulum stress-induced autophagy on cell survival. J Biol Chem 2007;282(7):4702-4710. doi:10.1074/jbc. M609267200.

[69] Lopes F, Keita ÅV, Saxena A, Reyes JL, Mancini NL, Al Rajabi A, et al. ERstress mobilization of death-associated protein kinase-1-dependent xenophagy counteracts mitochondria stress-induced epithelial barrier dysfunction. J Biol Chem 2018;293(9):3073-3087. doi:10.1074/ jbc.RA117.000809.

[70] Fiocchi C. IBD: the need for sweeping new concepts in pathogenesis and therapy. Curr Opin Gastroenterol 2017;33(4):219-221. doi:10.1097/MOG.0000000000000375.

[71] Zhong Q, Zhou B, Ann DK, Minoo P, Liu Y, Banfalvi A, et al. Role of endoplasmic reticulum stress in epithelial-mesenchymal transition of alveolar epithelial cells: effects of misfolded surfactant protein. Am J Respir Cell Mol Biol 2011;45(3):498-509. doi:10.1165/rcmb.201003470C.

[72] Baumgart DC, Sandborn WJ. Crohn's disease. Lancet 2012;380(9853): 1590-1605. doi:10.1016/S0140-6736(12)60026-9.

[73] Mu YP, Ogawa T, Kawada N. Reversibility of fibrosis, inflammation, and endoplasmic reticulum stress in the liver of rats fed a methionine-choline-deficient diet. Lab Invest 2010;90(2):245-256. doi:10.1038/labinvest.2009.123. 